\title{
Can Evaluation of Targeted Therapy in Oncology Be Improved by Means of ${ }^{18}$ F-FLT?
}

O ne of the most promising aspects of molecular imaging is its potential capacity to measure therapy effects long before morphologic changes are detected. The most frequently used PET tracer in oncology remains ${ }^{18} \mathrm{~F}$ FDG. However, despite its high sensitivity, this tracer has some major drawbacks, of which the generally low specificity is the main important limitation. Therefore, other, more specific tracers have been evaluated. One of the most promising and thoroughly studied radiopharmaceuticals is the proliferation marker $3^{\prime}$-deoxy- $3^{\prime}-{ }^{18} \mathrm{~F}$ fluorothymidine $\left({ }^{18} \mathrm{~F}-\mathrm{FLT}\right)$. Accumulation of ${ }^{18} \mathrm{~F}$-FLT in tumor cells has

See page 1559

been shown to be dependent on cellular thymidine kinase- 1 activity, the key enzyme and the limiting step of the pyrimidine salvage pathway of DNA synthesis, which is overexpressed in most tumor types (1). After monophosphorylation of ${ }^{18} \mathrm{~F}$-FLT by thymidine kinase- $1,{ }^{18} \mathrm{~F}$-FLT is intracellularly trapped. Because thymidine kinase-1 is functional only in the late G1- and S-phase of the cell cycle, ${ }^{18} \mathrm{~F}$ FLT uptake closely correlates to the amount of proliferating cells $(2,3)$.

Although ${ }^{18} \mathrm{~F}$-FLT uptake is generally lower than ${ }^{18} \mathrm{~F}-\mathrm{FDG}$ uptake, making it unlikely that ${ }^{18} \mathrm{~F}$-FLT will replace ${ }^{18} \mathrm{~F}-\mathrm{FDG}$ for staging purposes,

Received Jun. 4, 2010; revision accepted Jul. 2, 2010.

For correspondence or reprints contact: Felix M. Mottaghy, Department of Nuclear Medicine, RWTH Aachen University Hospital, Pauwelsstrasse 30, 52074 Aachen, Germany.

E-mail: fmottaghy@ukaachen.de

COPYRIGHT (C 2010 by the Society of Nuclear Medicine, Inc.

DOI: 10.2967/jnumed.110.079020 the higher specificity of this tracer and lower false-positive rate is a major advantage for tumor grading and early response assessment. The main cause of the limited specificity of ${ }^{18} \mathrm{~F}-\mathrm{FDG}$ is the high uptake in inflammatory cells, which cannot be differentiated from malignant cells. A much lower uptake in inflammatory tissue was shown for ${ }^{18} \mathrm{~F}-\mathrm{FLT}$ than for ${ }^{18} \mathrm{~F}$-FDG (4). However, the initial enthusiasm about the higher specificity of ${ }^{18} \mathrm{~F}$-FLT has been tempered by recent reports that ${ }^{18} \mathrm{~F}$ FLT uptake also occurs in granulomatous inflammatory lesions such as tuberculosis (5) and in reactive lymph nodes (6), being related to a high proliferation rate of macrophages and B-lymphocytes, respectively. Next to the inflammatory processes, which can be mistaken for tumors, transient inflammatory changes can occur inside a tumor as a reaction to therapy, inducing a temporarily increased ${ }^{18} \mathrm{~F}-\mathrm{FDG}$ uptake. Thereby, measurement of ${ }^{18} \mathrm{~F}-\mathrm{FDG}$ uptake could result in an underestimation of therapy response. Because inflammatory cells have a much lower proliferation rate, proliferation tracers such as ${ }^{18} \mathrm{~F}$-FLT will be less hampered by this phenomenon, and measurements of tracer uptake will more accurately reflect tumor response. A recently published study confirmed that a temporary rise in inflammatory cells after cyclophosphamide administration did not significantly influence ${ }^{18} \mathrm{~F}$-FLT uptake, whereas ${ }^{18}$ F-FDG uptake was temporary increased (7).

Besides the issue of inflammatory response, many new cancer-treatment agents induce cell-cycle arrest instead of tumor cell death and are not expected to lead to fast tumor regression. This issue makes measurements of cellular viability by ${ }^{18} \mathrm{~F}-\mathrm{FDG}$ theoretically less relevant, and the assessment of cellu- lar proliferation by ${ }^{18} \mathrm{~F}$-FLT might be a valid alternative. Disease-specific molecularly targeted agents increasingly replace the empiric combinations of cytotoxic agents from the past, because cytotoxic agents frequently lead to resistance and with each subsequent relapse the response rate will decrease (8). An example of the current targeted strategies is inhibition of the mammalian target of rapamycin (mTOR). Several analogs, such as temsirolimus (CCI-779; Wyeth), everolimus (RAD-001; Novartis), and deforolimus (AP23573;ArIAD and Merck), are being tested in clinical trials for treatment of mantle cell lymphoma, ovarian cancer, neuroendocrine carcinoma, and endometrial carcinoma $(8,9)$. mTOR is a regulator of cellular proliferation and acts through several targets. One of these targets is the messenger RNA encoding for the cyclin D1 protein, involved in cell-cycle regulation. Blocking mTOR leads to an inhibition of the translation of cyclin D1 messenger RNA to the cyclin D1 protein and provokes cell-cycle arrest in mid to late G1 (10), before the upregulation of thymidine kinase-1 in the S-phase, and thereby directly influencing ${ }^{18} \mathrm{~F}$-FLT uptake in the cell. As a result, imaging of proliferation with ${ }^{18} \mathrm{~F}-\mathrm{FLT}$ can directly measure the effect of mTOR inhibition and distinguish patients responding to mTOR inhibition from patients experiencing only the side effects of the therapy.

In half of all advanced ovarian cancers, p53 is mutated. This mutation is associated with a lack of response to cisplatin therapy, and as a result many of these patients have incurable disease (11). Previous studies showed that inhibition of mTOR blocks ovarian cancer cell proliferation and enhances the effect of cisplatin (12). ${ }^{18}$ F-FDG has been shown to be ineffective in predicting response to 
mTOR inhibition in patients with solid tumors (13). In this issue of The Journal of Nuclear Medicine, Aide et al. present an important preclinical study on ${ }^{18}$ F-FLT PET after mTOR inhibition in a cisplatin-resistant ovarian tumor model (14). Aide et al. aimed to evaluate ${ }^{18} \mathrm{~F}$-FLT PET during a daily administered everolimus therapy. ${ }^{18} \mathrm{~F}-\mathrm{FLT}$ uptake was correlated to bromodeoxyuridine uptake as a marker of cell proliferation and phosphorylation of ribosomal protein S6 as a downstream marker of mTOR activation. ${ }^{18} \mathrm{~F}$-FLT uptake decreased $2 \mathrm{~d}$ after initiation of treatment, with a more pronounced effect at day 7 of mTOR inhibition. Correlative immunohistochemistry showed a marked decrease in pS6 activity and bromodeoxyuridine incorporation corresponding to the decreased level of ${ }^{18}$ F-FLT uptake. In this preclinical feasibility study no correlation with outcome, or in other words the value of ${ }^{18} \mathrm{~F}$-FLT for the prediction of response, was studied. However, this aspect of molecular imaging, especially, should be further evaluated to really demonstrate its usefulness in daily routine.

A recently published study described ${ }^{18} \mathrm{~F}-\mathrm{FDG}$ and ${ }^{18} \mathrm{~F}-\mathrm{FLT}$ imaging after a single dose of temsirolimus or cyclophosphamide in a mouse model of mantle cell lymphoma (7). ${ }^{18}$ F-FLT uptake decreased early after mTOR inhibition, in correlation with cyclin D1 expression, which dropped from day 1 until day 4 . However, on day 7 after mTOR inhibition a temporary rise was observed in ${ }^{18}$ F-FLT uptake and cyclin D1. It is possible that still-viable tumor cells reenter the $\mathrm{S}$ phase after removal of the drug (half-life of temsirolimus is 9-17 h). Additionally, it is important to notice that ${ }^{18} \mathrm{~F}$-FLT is only slightly incorporated into DNA and that thymidine kinase-1 may be upregulated despite an inhibition of the DNA synthesis. ${ }^{18} \mathrm{~F}$ FLT uptake might also be stimulated by cellular repair mechanisms or the salvage pathway of the pyrimidine metabolism (15). An in vitro study observed an early increase in ${ }^{18} \mathrm{~F}$-FLT uptake $24 \mathrm{~h}$ after 5-fluorouracil due to blocking of the de novo pathway of the pyrimidine metabolism, thereby inducing the salvage pathway and redistributing nucleoside transporters to the plasma membrane (16). In the study of Aide et al. (14), no temporary ${ }^{18}$ F-FLT rise was observed during therapy. This observation is likely due to the permanent mTOR inhibition, as everolimus was administered daily in this study whereas the ${ }^{18} \mathrm{~F}$-FLT rise was observed $7 \mathrm{~d}$ after a single treatment.

Although a temporary increase in ${ }^{18}$ F-FLT signal after treatment should be taken into consideration, it is clear that the paper from Aide et al. supports the concept of early response assessment with ${ }^{18} \mathrm{~F}$-FLT. In this context, a recent paper reported an ${ }^{18} \mathrm{~F}-\mathrm{FLT}$ decrease after cytotoxic chemotherapy in patients with metastatic germ cell tumor (17). However, no significant differences were observed between histologic responders and nonresponders. In conclusion, the current evidence suggests that ${ }^{18} \mathrm{~F}$-FLT monitoring is more likely to be successful in patients undergoing cytostatic therapy. Of course, this possibility will have to be evaluated in larger clinical trials for different tumor entities.

\section{Marijke De Saint-Hubert \\ Lieselot Brepoels \\ Department of Nuclear Medicine \\ University Hospital Gasthuisberg Leuven \\ Leuven, Belgium}

Felix M. Mottaghy

Department of Nuclear Medicine RWTH Aachen University Hospital Aachen, Germany

Department of Nuclear Medicine Maastricht University Medical Center Maastricht, The Netherlands

\section{REFERENCES}

1. Seitz U, Wagner M, Neumaier B, et al. Evaluation of pyrimidine metabolising enzymes and in vitro uptake of $3^{\prime}-\left[{ }^{18} \mathrm{~F}\right]$ fluoro-3'-deoxythymidine $\left(\left[{ }^{18} \mathrm{~F}\right]\right.$ FLT) in pancreatic cancer cell lines. Eur J Nucl Med Mol Imaging. 2002;29:1174-1181.

2. Leyton J, Latigo JR, Perumal M, Dhaliwal H, He Q, Aboagye EO. Early detection of tumor response to chemotherapy by $3^{\prime}$-deoxy- $3^{\prime}-\left[{ }^{18}\right.$ F $]$ fluorothymidine positron emission tomography: the effect of cisplatin on a fibrosarcoma tumor model in vivo. Cancer Res. 2005;65:4202-4210.

3. Yap CS, Czernin J, Fishbein MC, et al. Evaluation of thoracic tumors with ${ }^{18} \mathrm{~F}$-fluorothymidine and ${ }^{18} \mathrm{~F}$ fluorodeoxyglucose-positron emission tomography. Chest. 2006;129:393-401.

4. Been LB, Suurmeijer AJ, Cobben DC, Jager PL, Hoekstra HJ, Elsinga PH. $\left[{ }^{18}\right.$ F $]$ FLT-PET in oncology: current status and opportunities. Eur J Nucl Med Mol Imaging. 2004;31:1659-1672.

5. Zhao S, Kuge Y, Kohanawa M, et al. Usefulness of ${ }^{11} \mathrm{C}$-methionine for differentiating tumors from granulomas in experimental rat models: a comparison with ${ }^{18} \mathrm{~F}$-FDG and ${ }^{18} \mathrm{~F}-\mathrm{FLT}$. $\mathrm{J} \mathrm{Nucl}$ Med. 2008;49:135-141.

6. Troost EG, Vogel WV, Merkx MA, et al. ${ }^{18}$ F-FLT PET does not discriminate between reactive and metastatic lymph nodes in primary head and neck cancer patients. J Nucl Med. 2007;48:726-735.

7. Brepoels L, Stroobants S, Verhoef G, De Groot T, Mortelmans L, De Wolf-Peeters C. ${ }^{18}$ F-FDG and ${ }^{18}$ F-FLT uptake early after cyclophosphamide and mTOR inhibition in an experimental lymphoma model. J Nucl Med. 2009;50:11021109.

8. Yap TA, Carden CP, Kaye SB. Beyond chemotherapy: targeted therapies in ovarian cancer. Nat Rev Cancer. 2009;9:167-181.

9. Duran I, Kortmansky J, Singh D, et al. A phase II clinical and pharmacodynamic study of temsirolimus in advanced neuroendocrine carcinomas. $\mathrm{Br} \mathrm{J}$ Cancer. 2006;95:1148-1154.

10. Costa LJ. Aspects of mTOR biology and the use of mTOR inhibitors in non-Hodgkin's lymphoma. Cancer Treat Rev. 2007;33:78-84.

11. Lavarino C, Pilotti S, Oggionni M, et al. p53 gene status and response to platinum/paclitaxel-based chemotherapy in advanced ovarian carcinoma. J Clin Oncol. 2000;18:3936-3945.

12. Mabuchi S, Altomare DA, Cheung M, et al. RAD001 inhibits human ovarian cancer cell proliferation, enhances cisplatin-induced apoptosis, and prolongs survival in an ovarian cancer model. Clin Cancer Res. 2007;13:4261-4270.

13. Ma WW, Jacene $H$, Song $D$, et al. $\left[{ }^{18} F\right]$ fluorodeoxyglucose positron emission tomography correlates with Akt pathway activity but is not predictive of clinical outcome during mTOR inhibitor therapy. J Clin Oncol. 2009;27:2697-2704.

14. Aide N, Kinross K, Cullinane C, et al. ${ }^{18} \mathrm{~F}-\mathrm{FLT}$ PET as a surrogate marker of drug efficacy during mTOR inhibition by everolimus in a preclinical cisplatin-resistant ovarian tumor model. J Nucl Med. 2010;51:1559-1564.

15. Mier W, Haberkorn U, Eisenhut M. $\left[{ }^{18} \mathrm{~F}\right] \mathrm{FLT}$ : portrait of a proliferation marker. Eur J Nucl Med Mol Imaging. 2002;29:165-169.

16. Dittmann H, Dohmen BM, Kehlbach R, et al. Early changes in $\left[{ }^{18} \mathrm{~F}\right] \mathrm{FLT}$ uptake after chemotherapy: an experimental study. Eur J Nucl Med Mol Imaging. 2002;29:1462-1469.

17. Pfannenberg C, Aschoff P, Dittmann H, et al. PET/ CT with ${ }^{18}$ F-FLT: does it improve the therapeutic management of metastatic germ cell tumors? J Nucl Med. 2010;51:845-853. 